\title{
Brane SUSY breaking and the gravitino mass
}

\author{
Noriaki Kitazawa \\ Department of Physics, Tokyo Metropolitan University, \\ Hachioji, Tokyo 192-0397, Japan \\ E-mail: noriaki.kitazawa@tmu.ac.jp
}

ABSTRACT: Supergravity models with spontaneously broken supersymmetry have been widely investigated over the years, together with some notable non-linear limits. Although in these models the gravitino becomes naturally massive absorbing the degrees of freedom of a Nambu-Goldstone fermion, there are cases in which the naive counting of degrees of freedom does not apply, in particular because of the absence of explicit gravitino mass terms in unitary gauge. The corresponding models require non-trivial de Sitter-like backgrounds, and it becomes of interest to clarify the fate of their Nambu-Goldstone modes. We elaborate on the fact that these non-trivial backgrounds can accommodate, consistently, gravitino fields carrying a number of degrees of freedom that is intermediate between those of massless and massive fields in a flat spacetime. For instance, in a simple supergravity model of this type with de Sitter background, the overall degrees of freedom of gravitino are as many as for a massive spin- $3 / 2$ field in flat spacetime, while the gravitino remains massless in the sense that it undergoes null-cone propagation in the stereographic picture. On the other hand, in the ten-dimensional USp(32) Type I Sugimoto model with "brane SUSY breaking", which requires a more complicated background, the degrees of freedom of gravitino are half as many of those of a massive one, and yet it somehow behaves again as a massless one.

KeYwords: Supersymmetry Breaking, D-branes, Supergravity Models

ARXIV EPRINT: 1802.03088 


\section{Contents}

1 Introduction 1

2 Supergravity models with non-linear supersymmetry 2

3 Massless propagation in de Sitter backgrounds 5

4 Massless gravitino in non-linear supersymmetry 9

5 Conclusions 14

\section{Introduction}

If supersymmetry [1-9] plays a role in the fundamental interactions, one is naturally led to Supergravity [10-14] and String Theory [15-21], where supersymmetry must be spontaneously broken at low energies. There is no general agreement, as of today, on the detailed dynamics of supersymmetry breaking in String Theory, and therefore it is important to explore further its model-independent realizations in the low-energy effective Supergravity. Non-linear realizations of supersymmetry are particularly interesting, because they encode information that is independent of the detailed fundamental dynamics.

The non-linear realization of global supersymmetry emerged very early, in the VolkovAkulov model [22], whose first application to supergravity models was discussed in [23-25]. This framework remains of great interest, and its coupling to Supergravity was recently reconsidered in [26-29], in the light of constrained superfields [30-33], and in the light of non-BPS D-branes [34, 35]. As was explained in [25], one must introduce mass terms for the gravitino and Nambu-Goldstone fermion and modify accordingly its supersymmetry transformation in order to eliminate a cosmological constant term to arrive at flat-space models. The systematics of these constructions, examined in detail in [36-39], led eventually to the no-scale models of [40], which also found a string realization in the presence of internal fluxes [41].

Typically, the gravitino becomes massive absorbing the degrees of freedom of a NambuGoldstone fermion, a phenomenon that becomes manifest in a unitary gauge, but in the ten-dimensional and six-dimensional orientifold models [42-51] with "brane supersymmetry breaking" [52-57] supersymmetry is non-linearly realized and no explicit gravitino mass term is allowed $[58,59]$, since the gravitino is a Majorana-Weyl fermion. ${ }^{1}$ On the other

\footnotetext{
1 "Brane supersymmetry breaking" is a way to break supersymmetry by brane configurations without tachyon instabilities. For example, the simultaneous presence of branes and anti-branes in order to break supersymmetry gives rise to tachyon instabilities corresponding to their pair annihilations. The combination of anti-D-branes and USp-type orientifold fixed planes is a typical configuration of "brane supersymmetry breaking" in which no tachyon instability appears, because these two objects do not annihilate and the system can be stable. See the review article in [53-57] for further details.
} 
hand, these models require non-trivial backgrounds, since they include a dilaton-dependent cosmological term $[60,61]$. Since no gravitino mass term emerges in a unitary gauge, the role of the Nambu-Goldstone fermion may appear confusing. Our aim here is to elaborate on the fate of the degrees of freedom of Nambu-Goldstone fermions in this second class of models. There are some interesting aspects in this story, since the curved spacetime is crucial in a proper account of the related degrees of freedom.

Much effort was devoted, over the years, to providing suitable definitions of masses and degrees of freedom in de Sitter spacetime, in particular in [62-66]. A highlight of these works is that null-cone propagation takes naturally the place of masslessness in flat space time [62], and the correspondence is illuminated by the special choice of "symmetric coordinates" described in [67]. The detailed investigation of this criterion of masslessness in the second class of models will lead us to a variant that is more suitable for "brane supersymmetry breaking", a "cosmological" criterion of masslessness in flat slicing coordinates. The new criterion applies, in particular, to the ten-dimensional Sugimoto model of [52] whose background is not exactly de Sitter spacetime. As we shall see, the gravitino in the Sugimoto model remains surprising massless in this sense, although it does absorb the degrees of freedom of a Nambu-Goldstone fermion.

In the next section we recall briefly some aspects of non-linear supersymmetry and the super-Higgs mechanism. As a warm up, we compare two simple models of pure supergravity without matter fields with and without a gravitino mass term. The latter model is an explicit realization of the equations and constraints for a massless spin- $3 / 2$ field that were described in [62] without specifying the action. This is an instructive step, since the low-energy supergravity action of the Sugimoto model is also an example of non-linear supersymmetry without a gravitino mass term. In section 3 we discuss the massless condition for fermions in a de Sitter spacetime. We briefly review the role of null-cone propagation in the symmetric coordinate system and conformal invariance, investigating explicitly a simple model involving a spinor field. We then propose a new masslessness criterion for fermion fields, which refers to the "rest mass" contribution to the "cosmological" energy density in a flat slicing coordinate system for a de Sitter spacetime. In section 4 we demonstrate that, in a simple model without gravitino mass term that will be introduced in the next section, the gravitino remains massless in this sense, displaying its null-cone propagation and also applying our new criterion. We then show that the gravitino in the Sugimoto model remains massless, in the sense specified above, via our new criterion, which applies insofar as the background can be understood as a cosmological evolution. The overall number of degrees of freedom of the massless gravitino is the sum of those of a massless gravitino in flat spacetime and a Nambu-Goldstone fermion, which is half as many of those of a massive spin- $3 / 2$ field. The last section contains some concluding remarks.

\section{Supergravity models with non-linear supersymmetry}

Let us begin by considering pure supergravity

$$
\mathcal{L}_{\text {pure }}=\frac{1}{2 \kappa^{2}} e \mathcal{R}(e, \omega)-e \frac{1}{2} \bar{\psi}_{\mu} \Gamma^{\mu \rho \nu} D_{\rho} \psi_{\nu}+\mathcal{O}\left(\left(\psi_{\mu}\right)^{4}\right),
$$


while leaving aside higher powers in the Majorana gravitino field. Here, we work sketchily in a generic spacetime dimension $D \geq 4$, which allows Majorana fermions, at the cost of leaving aside some members of the supergravity multiplet. In this paper we follow the conventions of [68]. This Lagrangian is invariant, up to a total divergence and higher powers of gravitino, under the transformations

$$
\begin{aligned}
\delta e^{m}{ }_{\mu} & =-\frac{1}{2} \kappa \bar{\psi}_{\mu} \Gamma^{m} \epsilon, \\
\delta \psi_{\mu} & =\frac{1}{\kappa} D_{\mu} \epsilon,
\end{aligned}
$$

whose parameter is the Majorana spinor $\epsilon$. The covariant derivative of the spinor field involves the spin connection $\omega$ and reads

$$
D_{\mu} \epsilon=\left(\partial_{\mu}+\frac{1}{4} \omega_{\mu}^{m n} \Gamma_{m n}\right) \epsilon
$$

where local Lorentz indices are denoted by Latin letters. Let us now introduce a NambuGoldstone fermion field $\theta$ that provides a non-linear realization of supersymmetry

$$
\mathcal{L}_{\mathrm{NL}}=-e 2 f^{2}-e \frac{1}{2} \bar{\theta} \Gamma^{\mu} D_{\mu} \theta+\mathcal{O}\left((\theta)^{4}\right)
$$

whose lowest-order coupling to the gravitino

$$
\mathcal{L}_{\text {current }}=e \kappa \bar{\psi}_{\mu} S^{\mu}
$$

involves the supersymmetry current

$$
S_{\mu}=-f \Gamma_{\mu} \theta
$$

The dimensionful quantity $f$ defines the scale of supersymmetry breaking and the NambuGoldstone fermion field transforms, to lowest order, according to

$$
\delta \theta=f \epsilon
$$

The total Lagrangian $\mathcal{L}_{\text {pure }}+\mathcal{L}_{\mathrm{NL}}+\mathcal{L}_{\text {current }}$

$$
\mathcal{L}=\frac{1}{2 \kappa^{2}} e \mathcal{R}(e, \omega)-e \frac{1}{2} \bar{\psi}_{\mu} \Gamma^{\mu \rho \nu} D_{\rho} \psi_{\nu}-e \frac{1}{2} \bar{\theta} \Gamma^{\mu} D_{\mu} \theta+e \kappa \bar{\psi}_{\mu} S^{\mu}-e 2 f^{2}
$$

is invariant under these supersymmetry transformations, up to higher terms that we are not tracking here $[25,69]$. This example involves on purpose no gravitino mass term in the unitary gauge $\theta=0$. Note that this model contains a positive cosmological constant term, so that one is naturally led to consider a background de Sitter spacetime. The lesson of these old considerations is that the absence of the mass term does not necessarily imply that the gravitino is a simple massless spin $-3 / 2$ field, which conforms to the fate of the degrees of freedom introduced by Nambu-Goldstone fermion. The purpose of this paper is to elaborate on what happens to the gravitino and the Nambu-Goldstone fermion in more complicated models of broken Supersymmetry. 
Let us now turn to another pure supergravity model with a gravitino mass and a negative cosmological constant, so that

$$
\mathcal{L}_{\text {pure(massive) }}=\frac{1}{2 \kappa^{2}} e \mathcal{R}(e, \omega)-e \frac{1}{2} \bar{\psi}_{\mu} \Gamma^{\mu \rho \nu} D_{\rho} \psi_{\nu}+e \frac{1}{2} m \bar{\psi}_{\mu} \Gamma^{\mu \nu} \psi_{\nu}+e C,
$$

where

$$
C=\frac{2(D-1)}{(D-2)} \frac{m^{2}}{\kappa^{2}}
$$

The gauge transformations of this model are different, and include the contributions

$$
\begin{aligned}
\delta e_{\mu}^{m} & =-\frac{1}{2} \kappa \bar{\psi}_{\mu} \Gamma^{m} \epsilon, \\
\delta \psi_{\mu} & =\frac{1}{\kappa}\left(D_{\mu} \epsilon+\frac{1}{D-2} m \Gamma_{\mu} \epsilon\right) .
\end{aligned}
$$

Making the Lagrangian $\mathcal{L}_{\mathrm{NL}}+\mathcal{L}_{\text {current }}$ invariant under the new gauge transformation requires the introduction of a mass term for the Nambu-Goldstone fermion [70, 71],

$$
\mathcal{L}_{\text {mass }}=-e \frac{1}{2} \frac{D}{D-2} m \bar{\theta} \theta
$$

The portion of the total Lagrangian that we are focussing on, $\mathcal{L}_{\text {pure(masive) }}+\mathcal{L}_{\mathrm{NL}}+\mathcal{L}_{\text {current }}+$ $\mathcal{L}_{\text {mass }}$, is then invariant under the new gauge transformation, up to higher order terms or contributions involving the other members of the gravity or matter multiplets. In particular, a model without cosmological constant term would require tuning $2 f^{2}=C$, and thus linking the mass parameters of the gravitino and the Nambu-Goldstone fermion, $m$, to the supersymmetry breaking scale $f$. In a model of this type admitting a flat spacetime background the gravitino has a mass term in the unitary gauge $\theta=0$, and in fact it is a conventional massive spin- $3 / 2$ field.

Models that are similar to some extent to those in eq. (2.9) are realized in orientifolds with brane supersymmetry breaking, and the ten-dimensional Sugimoto model is the simplest example in this class. At tree level the supersymmetry present in the original type IIB closed string sector is halved by the orientifold projection, as in the type-I superstring, while in the open sector supersymmetry is completely broken, or non-linearly realized. The low-energy effective Lagrangian of the Sugimoto model was discussed in detail in [58, 59]. In Einstein frame and in unitary gauge it combines open and closed contributions,

$$
\mathcal{L}=\mathcal{L}_{\text {closed }}+\mathcal{L}_{\text {open }}
$$

where

$$
\begin{aligned}
\mathcal{L}_{\text {closed }}= & \frac{1}{2 \kappa_{10}^{2}} e\left\{R-\frac{1}{2} \partial^{\mu} \phi \partial_{\mu} \phi-\frac{1}{12} e^{-\phi} F^{\mu \nu \rho} F_{\mu \nu \rho}-\frac{1}{2} \bar{\psi}_{\mu} \Gamma^{\mu \rho \nu} D_{\rho} \psi_{\nu}-\frac{1}{2} \bar{\lambda} \Gamma^{\mu} D_{\mu} \lambda\right. \\
& \left.-\frac{1}{2 \sqrt{2}} \bar{\psi}_{\mu} \Gamma^{\nu} \Gamma^{\mu} \lambda \partial_{\nu} \phi-\frac{1}{48} e^{-\frac{1}{2} \phi}\left[\bar{\psi}_{[\mu} \Gamma^{\mu} \Gamma^{\rho \sigma \tau} \Gamma^{\nu} \psi_{\nu]}-\sqrt{2} \bar{\psi}_{\mu} \Gamma^{\rho \sigma \tau} \Gamma^{\mu} \lambda\right] F_{\rho \sigma \tau}\right\}
\end{aligned}
$$


describes the contribution from the closed sector, while

$$
\begin{aligned}
\mathcal{L}_{\text {open }}= & \frac{1}{2 \kappa_{10}^{2}} e\left\{-e^{\frac{1}{2} \phi} \frac{1}{2 g^{2}} \operatorname{tr}\left(F^{\mu \nu} F_{\mu \nu}\right)-\frac{1}{g^{2}} \operatorname{tr}\left(\bar{\chi} \Gamma^{\mu} D_{\mu} \chi\right)\right. \\
& \left.-2 \alpha_{E} e^{\frac{3}{2} \phi}+e^{-\frac{1}{2} \phi} \frac{1}{24 g^{2}} \operatorname{tr}\left(\bar{\chi} \Gamma^{\rho \sigma \tau} \chi\right) F_{\rho \sigma \tau}\right\}
\end{aligned}
$$

describes the contribution from open sector. Beginning from the closed sector, $\phi$ is the dilaton field, $F_{\mu \nu \rho}$ is the field strength tensor of an antisymmetric tensor field (a RamondRamond field), $\psi_{\mu}$ is the gravitino field and $\lambda$ is the dilatino field. Turning now to the open sector, $F_{\mu \nu}$ is the field strength of the $\operatorname{USp}(32)$ gauge fields and $\chi$ is a spinor field belonging to the traceless anti-symmetric representation of the $\operatorname{USp}(32)$ gauge group. On the other hand the Nambu-Goldstone fermion, which has been set to zero in the unitary gauge, would arise from the corresponding symplectic trace. The gauge coupling and "cosmological constant" are $g^{2}=2 / \alpha^{\prime}$ and $\alpha_{E}=64 T_{9} \kappa_{10}^{2}$, respectively, where $T_{9}$ is the tension of anti-D9-brane.

In the following we also set to zero the fields $F_{\mu \nu \rho}, F_{\mu \nu}$ and $\chi$, since they do not play any role in the non-trivial backgrounds of interest that are required by the dilaton-dependent cosmological term and in the corresponding realization of supersymmetry breaking. The model thus reduces to

$$
\mathcal{L}=\frac{1}{2 \kappa_{10}^{2}} e\left\{R-\frac{1}{2} \partial^{\mu} \phi \partial_{\mu} \phi-\frac{1}{2} \bar{\psi}_{\mu} \Gamma^{\mu \rho \nu} D_{\rho} \psi_{\nu}-\frac{1}{2} \bar{\lambda} \Gamma^{\mu} D_{\mu} \lambda-\frac{1}{2 \sqrt{2}} \bar{\psi}_{\mu} \Gamma^{\nu} \Gamma^{\mu} \lambda \partial_{\nu} \phi-2 \alpha_{E} e^{\frac{3}{2} \phi}\right\} .
$$

Now the field equation of the Nambu-Goldstone fermion yields

$$
\Gamma^{\mu} \psi_{\mu}=-\frac{3}{\sqrt{2}} \lambda
$$

which identifies the dilatino and the gamma trace of the gravitino. We see that there is no mass term for gravitino, and therefore this model belongs to the same class as the model of eq. (2.9). There is an additional reason for the absence of a gravitino mass term: in this ten-dimensional setting this field is a Majorana-Weyl fermion field, for which the mass term simply does not exist. The goal of this paper is to elaborate on what happens to the gravitino in this more complicated model, in the presence of non-trivial background fields.

\section{Massless propagation in de Sitter backgrounds}

It is well known that one can define a $D$-dimensional de Sitter spacetime starting from the quadratic constraint

$$
\eta_{\alpha \beta} \xi^{\alpha} \xi^{\beta}=-\left(\xi^{0}\right)^{2}+\left(\xi^{1}\right)^{2}+\cdots+\left(\xi^{D}\right)^{2}=1 / H^{2} .
$$

Here $\xi^{\alpha}$ with $\alpha=0,1, \cdots, D$ are ambient coordinates and $1 / H$ is the de Sitter radius, which is related to the cosmological constant $\Lambda>0$ according to $\Lambda=(D-1)(D-2) H^{2} / 2 \kappa^{2}$. The 
curvature tensors are described by the metric tensor $g_{\mu \nu}$, independently of the coordinate system, as

$$
R_{\mu \sigma \nu}^{\rho}=H^{2}\left(\delta_{\sigma}^{\rho} g_{\mu \nu}-\delta_{\nu}^{\rho} g_{\mu \sigma}\right), \quad R_{\mu \nu}=R_{\mu \rho \nu}^{\rho}=(D-1) H^{2} g_{\mu \nu},
$$

with $\mu, \nu, \rho, \sigma=0,1, \cdots, D-1$, and the scalar curvature is a constant, with $R=D(D-$ 1) $H^{2}$. In this paper use two concrete coordinate systems, the symmetric coordinates and the flat slicing coordinates.

The symmetric coordinates [67], the result from a stereographic projection of the generalized hyperboloid of eq. (3.1) to $D$-dimensional flat spacetime, defined via

$$
\xi^{\mu}=\frac{x^{\mu}}{1+s}, \quad \xi^{D}=\frac{1-s}{1+s} \frac{1}{H},
$$

where

$$
s=\frac{H^{2}}{4} \eta_{\mu \nu} x^{\mu} x^{\nu}
$$

In this fashion the metric tensor takes the form

$$
g_{\mu \nu}=\Omega^{2} \eta_{\mu \nu}
$$

with $\Omega=(1+s)^{-1}$, while

$$
e_{\mu}^{m}=\Omega \delta_{\mu}^{m}, \quad e_{m}^{\mu}=\Omega^{-1} \delta_{m}^{\mu}
$$

is a corresponding vielbein. The Christoffel symbols and the spin connection are then

$$
\begin{aligned}
\Gamma_{\mu \nu}^{\rho} & =\frac{\Omega H^{2}}{2}\left[x^{\rho} \eta_{\mu \nu}-x_{\mu} \delta_{\nu}^{\rho}-x_{\nu} \delta_{\mu}^{\rho}\right], \\
\frac{1}{4} \omega_{\mu}{ }^{m n} \Gamma_{m n} & =-\Omega \frac{H^{2}}{4} \gamma_{\mu \nu} x^{\nu},
\end{aligned}
$$

respectively, where

$$
\Gamma^{\mu}=\gamma^{m} e_{m}^{\mu}=\Omega^{-1} \gamma^{\mu}, \quad\left\{\gamma^{m}, \gamma^{n}\right\}=2 \eta^{m n}
$$

Since this coordinate system yields a conformally flat metric, it is a convenient choice to investigate null-cone propagation and conformal covariance of the field equations.

The more conventional flat slicing coordinates of de Sitter spacetime are defined as

$$
\xi^{0}=\frac{1}{H} \sinh \left(H x^{0}\right)+\frac{H}{2} r^{2} e^{H x^{0}}, \quad \xi^{i}=x^{i} e^{H x^{0}}, \quad \xi^{D}=\frac{1}{H} \cosh \left(H x^{0}\right)-\frac{H}{2} r^{2} e^{H x^{0}},
$$

where $r^{2}=\delta^{i j} x^{i} x^{j}$ with $i, j=1, \cdots, D-1$. The metric is then

$$
d s^{2}=g_{\mu \nu} d x^{\mu} d x^{\nu}=-\left(d x^{0}\right)^{2}+e^{2 H x^{0}} \delta^{i j} d x^{i} d x^{j},
$$

and a corresponding vielbein is

$$
e_{\mu}^{m}=\operatorname{diag}\left(1, e^{H x^{0}}, e^{H x^{0}}, \cdots\right) .
$$


Christoffel symbols and spin connection are now

$$
\Gamma_{i j}^{0}=H g_{i j}, \quad \Gamma_{0 j}^{i}=\Gamma_{j 0}^{i}=H g_{j}^{i}, \quad \text { others }=0,
$$

and

$$
\omega_{\mu}{ }^{0 \bar{m}}=-\omega_{\mu}{ }^{\bar{m} 0}=H e_{\mu}{ }^{\bar{m}}, \quad \text { others }=0,
$$

respectively, where $\bar{m}=1,2, \cdots, D-1$. As is well known, this coordinate system covers half of the whole de Sitter spacetime, the portion where space expands in time, which affords a natural cosmological interpretation.

In a Minkowski spacetime the Casimir operator $\eta_{\mu \nu} \hat{p}^{\mu} \hat{p}^{\nu}$ of Poincaré group, where $\hat{p}^{\mu}$ is the generator of spacetime translation, is used to define the mass of the field. Since there is no such Casimir operator in the de $\operatorname{Sitter} \operatorname{SO}(D, 1)$ group, there is no natural way to define the mass of fields. Still, null-cone propagation remains a convincing criterion for masslessness.

Let us now briefly recall the massless criterion via null-cone propagation in the symmetric coordinate system [62]. To this end, let us consider a simple spin- $1 / 2$ spinor field in de Sitter background, for which

$$
\mathcal{L}_{\text {spin } 1 / 2}=-e \bar{\Psi} \Gamma^{\mu} D_{\mu} \Psi
$$

The field equation

$$
\Gamma^{\mu} D_{\mu} \Psi=0
$$

can be rewritten as

$$
\Gamma^{\mu} D_{\mu} \Psi=\Omega^{-\frac{D+1}{2}} \gamma^{\mu} \partial_{\mu} \psi=0 .
$$

where $\psi$ is the rescaled field defined by

$$
\Psi=\Omega^{w_{1 / 2}} \psi
$$

with the conformal weight of the spinor field $w_{1 / 2}=(1-D) / 2$. One is thus led to null-cone propagation, and in this respect the spinor field can be considered a massless field in de Sitter background. A similar conclusion can be reached starting from the second-order field equation.

$$
\Gamma^{\mu} D_{\mu} \Gamma^{\nu} D_{\nu} \Psi=\left(g^{\mu \nu} D_{\mu} D_{\nu}-\frac{D(D-1)}{4} H^{2}\right) \Psi=\Omega^{-\frac{3+D}{2}} \eta^{\mu \nu} \partial_{\mu} \partial_{\nu} \Psi=0
$$

which leads consistently to null-cone propagation and thus points again to the notion of a massless spinor field.

The action of this model is invariant under the de $\operatorname{Sitter} \operatorname{SO}(D, 2)$ conformal transformation. Since local Weyl invariance is enough for de Sitter conformal invariance [66], we show local Weyl invariance of this model. The Weyl transformation is defined as

$$
e_{\mu}{ }^{m} \longrightarrow \Omega_{W} e_{\mu}^{m}, \quad e_{m}{ }^{\mu} \longrightarrow \Omega_{W}^{-1} e_{\mu}{ }^{m}
$$

to be combined with

$$
\Psi \longrightarrow \Omega_{W}^{w_{1 / 2}} \Psi
$$


where $\Omega_{W}$ is the local Weyl scaling factor. Using the definition of the gamma matrices in a non-trivial background, $\Gamma^{\mu}=\gamma^{m} e_{m}{ }^{\mu}$, and the transformation of spin connection

$$
\omega_{\mu}^{m n} \longrightarrow \omega_{\mu}^{m n}+\left(e^{m} e^{n \nu}-e^{n} e^{m \nu}\right) \partial_{\nu} \ln \Omega_{W}
$$

it is straightforward to see the invariance of the Lagrangian of eq. (3.15) provided one chooses the conformal weight $w_{1 / 2}=(1-D) / 2$. Since conformal invariance reflects the absence of a specific scale, it is natural to regard the spinor field as a massless field.

We now turn to propose a massless criterion for fermion fields in the flat slicing coordinate system, referring again to the above model of a spinor field. The explicit form of the field equation in the flat slicing coordinate is

$$
\partial_{0} \Psi+\frac{D-1}{2} H \Psi=0 .
$$

Here, we have also assumed that the field depends only on time, since we anticipate the use of some physical arguments related to the homogeneous expansion of the Universe. The important property of the solution of this equation is that it is not oscillatory,

$$
\Psi \propto e^{-\frac{D-1}{2} H x^{0}}
$$

and consequently does not contribute to the energy density $T_{00}$, where

$$
T_{\mu \nu}=\frac{1}{2}\left(\bar{\Psi} \Gamma_{\nu} D_{\mu} \Psi-\bar{\Psi} \overleftarrow{D}_{\mu} \Gamma_{\nu} \Psi\right)
$$

The explicit form of $T_{00}$ in our present setting

$$
T_{00}=\frac{1}{2}\left\{\Psi^{\dagger}\left(i \partial_{0} \Psi\right)-\left(i \partial_{0} \Psi^{\dagger}\right) \Psi\right\}
$$

clearly indicates that it could be non-vanishing for an oscillatory solution $\Psi \propto e^{-i m x^{0}}$ with "rest mass" $m$. Hence, the non-oscillatory behavior of the Fermi field can regarded as an indication of masslessness.

We can now propose a similar argument for the second-order field equation, anticipating some steps that will prove useful for the application to the gravitino in the next section. The explicit form of the second-order field equation is

$$
\partial_{0} \partial_{0} \Psi+(D-1) H \partial_{0} \Psi+\left(\frac{D-1}{2}\right)^{2} H^{2} \Psi=0 .
$$

Let us now perform the rescaling of the field as in eq. (3.18) with $\Omega=e^{H x^{0}}$. The reason for this choice of $\Omega$ is that the metric can be described, in conformal time $\eta$, as

$$
d s^{2}=\Omega^{2}\left(-d \eta^{2}+\delta^{i j} d x^{i} d x^{j}\right)
$$

with $\Omega=1 /|H \eta|=e^{H x^{0}}$, so that this setting compares naturally with eq. (3.5). The field equation now takes the simple form

$$
\partial_{0} \partial_{0} \psi=0
$$

If we assume that a solution should be finite in the limit of $x^{0} \rightarrow \infty, \psi$ should be a constant, which is consistent with eq. (3.24). In this respect, a constant solution for the rescaled field in the flat slicing coordinate system can be a criterion of masslessness. 


\section{Massless gravitino in non-linear supersymmetry}

Consider the model of eq. (2.9) in the background de Sitter spacetime required by the cosmological constant $2 f^{2}$. The field equations of the gravitino and the Nambu-Goldstone fermion are

$$
\begin{aligned}
\Gamma^{\mu \rho \nu} D_{\rho} \psi_{\nu}+\kappa f \Gamma^{\mu} \theta & =0, \\
\Gamma^{\mu} D_{\mu} \theta-\kappa f \Gamma^{\mu} \psi_{\mu} & =0 .
\end{aligned}
$$

In the unitary gauge $\theta=0$, these equations reduce to one field equation with one constraint:

$$
\Gamma^{\mu \rho \nu} D_{\rho} \psi_{\nu}=0, \quad \Gamma^{\mu} \psi_{\mu}=0,
$$

the combination that was discussed as a model of null-cone propagation without de Sitter conformal invariance in [62]. Applying $\Gamma_{\mu}$ to the field equation gives another constraint

$$
D_{\mu} \psi^{\mu}=0
$$

and if $D \neq 2$ the field equation becomes

$$
\Gamma^{\mu} D_{\mu} \psi_{\nu}=0
$$

In the symmetric coordinate system the explicit form of this field equation for the rescaled field

$$
\psi_{\mu} \longrightarrow \Omega^{w_{3 / 2}} \psi_{\mu}
$$

with $w_{3 / 2}=(3-D) / 2$, is now

$$
\Omega^{-\frac{D-1}{2}} \gamma^{\mu} \partial_{\mu} \psi_{\nu}-\Omega^{-\frac{D-3}{2}} \frac{H^{2}}{2}\left(\gamma_{\nu} x^{\mu} \psi_{\mu}-x_{\nu} \gamma^{\mu} \psi_{\mu}\right)=0
$$

Eliminating $\gamma^{\mu} \psi_{\mu}$ and $x^{\mu} \psi_{\mu}$ using the explicit forms of the two constraints

$$
\gamma^{\mu} \psi_{\mu}=0, \quad \partial_{\mu} \psi^{\mu}=\Omega \frac{D H^{2}}{4} x^{\mu} \psi_{\mu}
$$

yields finally the field equation

$$
\gamma^{\mu} \partial_{\mu} \psi_{\nu}-\frac{2}{D} \gamma_{\nu} \partial_{\mu} \psi^{\mu}=0
$$

This is a well-known conformally covariant field equation. It was described in [62], and was derived in [72] in $D=4$. The difference with respect to the massless Rarita-Schwinger equation lies in the special value of the coefficient of the second term, which would be one for the massless Rarita-Schwinger equation rather than $2 / D$. Note that one can not take a naive flat limit $H \rightarrow 0$ in this conformally covariant field equation, since in the process to obtain it we had to perform a division by $H^{2}$ in order to eliminate the $x^{\mu} \psi_{\mu}$ term. As a result, the number of degrees of freedom of this spin- $3 / 2$ field undergoes a discontinuity in moving between flat and de Sitter spacetimes. 
Acting on eq. (4.9) with the "flat" divergence $\partial^{\nu}$ now gives, for $D \neq 2$,

$$
\gamma^{\mu} \partial_{\mu}\left(\partial_{\nu} \psi^{\nu}\right)=0
$$

so that the component $\partial_{\nu} \psi^{\nu}$ carrying spin- $1 / 2$ degrees of freedom propagates on the null cone. As a result, the other components of the field $\tilde{\psi}_{\mu}$, with $\partial_{\mu} \tilde{\psi}^{\mu}=0$ (and also $\gamma^{\mu} \tilde{\psi}_{\mu}=0$ ) satisfy the field equation

$$
\gamma^{\mu} \partial_{\mu} \tilde{\psi}_{\nu}=0
$$

which means again null-cone propagation. Therefore, the gravitino of this model can be regarded as massless in de Sitter spacetime, although its degrees of freedom combine those of a massless spin- $3 / 2$ field and of a massless spin- $1 / 2$ field in a flat Minkowski background. Let us stress that this is a different state of affairs from the "partially massless field" in [73].

In the flat slicing coordinate system, under the assumption that the field depends only on time, the second order field equation before rescaling the field

$$
\Gamma^{\mu} D_{\mu} \Gamma^{\nu} D_{\nu} \psi_{\rho}=g^{\mu \nu} D_{\mu} D_{\nu} \psi_{\rho}-\frac{1}{2} R_{\rho \mu \nu}^{\lambda} \Gamma^{\mu \nu} \psi_{\lambda}-\frac{1}{4} R \psi_{\rho}=0
$$

takes the form

$$
\begin{aligned}
\partial_{0} \partial_{0} \psi_{0}+(D-1) H \partial_{0} \psi_{0}+\frac{(D-5)(D-1)}{4} H^{2} \psi_{0} & =0 \\
\partial_{0} \partial_{0} \psi_{i}+(D-3) H \partial_{0} \psi_{i}+\frac{(D-5)(D-1)}{4} H^{2} \psi_{i}+H^{2} \Gamma_{0 i} \psi_{0}-H^{2} \Gamma_{i}{ }^{\lambda} \psi_{\lambda} & =0 .
\end{aligned}
$$

The constraint $D_{\mu} \psi^{\mu}=0$ now reads

$$
\partial_{0} \psi_{0}-\frac{1}{2} H \Gamma_{0} \Gamma^{i} \psi_{i}+(D-1) H \psi_{0}=0,
$$

and a simple solution of the above constraint and $\Gamma^{\mu} \psi_{\mu}=0$ is

$$
\psi_{0}=0, \quad \Gamma^{i} \psi_{i}=0 .
$$

In this fashion the second-order field equation becomes

$$
\partial_{0} \partial_{0} \psi_{i}+(D-3) H \partial_{0} \psi_{i}+\left(\frac{D-3}{2}\right)^{2} H^{2} \psi_{i}=0
$$

This equation is very similar to eq. (3.27) for the spinor field. Rescaling the field according to

$$
\psi_{i} \longrightarrow \Omega^{w_{3 / 2}} \psi_{i}
$$

where $\Omega=e^{H x^{0}}$ and $w_{3 / 2}=(3-D) / 2$ is the conformal weight of vector-spinor field, the field equation takes finally the very simple form

$$
\partial_{0} \partial_{0} \psi_{i}=0
$$

If we add the reasonable assumption that the solution should be finite in the limit of $x^{0} \rightarrow \infty, \psi_{i}$ can only be a constant. This means that gravitino is massless, exactly as was 
the case for the spinor field in previous section. The energy-momentum tensor of the field before rescaling can be recast in the form

$$
T_{\mu \nu}=\frac{1}{4} g_{\rho \sigma}\left(\bar{\psi}^{\rho} \Gamma_{\nu} D_{\mu} \psi^{\sigma}-\bar{\psi}^{\rho} \overleftarrow{D}_{\mu} \Gamma_{\nu} \psi^{\sigma}\right)-\frac{1}{4}\left(\bar{\psi}^{\rho} \Gamma_{\nu} D_{\rho} \psi_{\mu}-\bar{\psi}_{\mu} \overleftarrow{D}_{\rho} \Gamma_{\nu} \psi^{\rho}\right)
$$

and the explicit form of $T_{00}$ in our present setting is

$$
T_{00}=\frac{1}{4} g_{i j}\left\{\left(\psi^{i}\right)^{\dagger}\left(i \partial_{0} \psi^{j}\right)-\left(i \partial_{0}\left(\psi^{i}\right)^{\dagger}\right) \psi^{j}\right\} .
$$

We see that this energy density function vanishes for the non-oscillatory solutions, as pertains to fields with a vanishing "rest mass".

We are now ready to investigate the behavior of the gravitino in the more complicated Sugimoto model. The relevant starting point was already introduced at the end of section 2, in eqs. (2.18) and (2.19). A time-dependent background, which can be interpreted as a cosmological evolution, was first obtained in $[60,61]$ starting from the ansatz

$$
d s^{2}=-e^{2 B}\left(d x^{0}\right)^{2}+e^{2 A} \delta^{i j} d x^{i} d x^{j},
$$

for the metric, where $A$ and $B$ are functions only of $x^{0}$. The gauge condition $B=-3 \phi / 4$ which corresponds to a convenient choice for the time coordinate reduces the equations for the background to

$$
\left\{\begin{array}{l}
\ddot{\phi}+(9 \dot{A}-\dot{B}) \dot{\phi}=-3, \\
8 \ddot{A}-8 \dot{A} \dot{B}+36(\dot{A})^{2}+\frac{1}{4}(\dot{\phi})^{2}=1, \\
36(\dot{A})^{2}-\frac{1}{4}(\dot{\phi})^{2}=1,
\end{array}\right.
$$

where dots indicate derivatives with respect to the dimensionless time $\tau=\sqrt{\alpha_{E}} x^{0}$. The solution then reads

$$
\left\{\begin{array}{l}
A=A_{0}+\frac{1}{18} \ln \tau+\frac{1}{16} \tau^{2}, \\
\phi=\phi_{0}+\frac{2}{3} \ln \tau-\frac{2}{4} \tau^{2} .
\end{array}\right.
$$

Notice that $\Omega=e^{A}$ can be regarded as the scale factor of cosmological expansion, while $\tau$ defines implicitly the cosmic time via

$$
d t=e^{-\frac{3}{4} \phi} \sqrt{\alpha_{E}} d \tau .
$$

This result of $[60,61]$ is a special instance of a class of solutions for Einstein gravity minimally coupled to a scalar field in the presence of an exponential potential proportional to $\exp (\gamma \phi)$, which were studied in detail in [74]. The exponent selected by String Theory for "brane supersymmetry breaking", $\gamma=3 / 2$ in the Einstein frame, has the key property of separating two regions of solutions with widely different behavior. As pointed out in [7577], this value marks the onset of the "climbing phenomenon", according to which the scalar field has no other option, when emerging from the initial singularity, than climbing up the potential before reaching a turning point and starting its descent. This is important, since 
this dynamics sets naturally an upper bound on the string coupling (although there are no indications of a similar bound on $\alpha^{\prime}$ corrections [78]), and suggests that the resulting descent could help one model the onset of inflation. A climbing phase could provide the impulse to start inflation [79-91], and in general an early fast-roll would introduce a lowfrequency cut in the primordial power spectrum of scalar perturbations. This option was explored over the years in different contexts [92-111], but it arguably obtains, in "brane supersymmetry breaking", an enticing input from String Theory. There are also some signs, away from the Galactic plane, of an encouraging comparison with the lack of power apparently present in the low- $\ell$ CMB [112-115].

There is an important link with the preceding case, since close to the turning point for the climbing scalar field, where $\dot{\phi}=0$ and $\ddot{A}=0$, the Universe in this case bears some similarities with de Sitter spacetime with temporally constant $\dot{A}$ in $\Omega=e^{A}$. The Christoffel symbols and the spin connection for this background are

$$
\Gamma_{i j}^{0}=\sqrt{\alpha_{E}} \dot{A} e^{2 A-2 B} \delta_{i j}, \quad \Gamma_{00}^{0}=\sqrt{\alpha_{E}} \dot{B}, \quad \Gamma_{0 j}^{i}=\Gamma_{j 0}^{i}=\sqrt{\alpha_{E}} \dot{A} \delta_{j}^{i}, \quad \text { others }=0,
$$

and

$$
\omega_{\mu}^{0 \bar{m}}=-\omega_{\mu}^{\bar{m} 0}=e^{-B} \sqrt{\alpha_{E}} \dot{A} e_{\mu}^{\bar{m}}, \quad \text { others }=0,
$$

respectively. The curvature tensors are described as

$$
\begin{aligned}
R_{\nu \rho \sigma}^{\mu}= & \alpha_{E}\left[\delta_{0}^{\mu}\left(\delta_{\rho}^{0} \eta_{\nu \sigma}-\delta_{\sigma}^{0} \eta_{\nu \rho}\right)(\ddot{A}-\dot{A} \dot{B}) e^{2 A-2 B}\right. \\
& +\delta_{\nu}^{0}\left(\delta_{\rho}^{0} \delta_{\sigma}^{\mu}-\delta_{\sigma}^{0} \delta_{\rho}^{\mu}\right)\left(\ddot{A}+\dot{A}^{2}-\dot{A} \dot{B}-\dot{A}^{2} e^{2 A-2 B}\right) \\
& \left.+\left(\delta_{\rho}^{\mu} \eta_{\nu \sigma}-\delta_{\sigma}^{\mu} \eta_{\nu \rho}\right) \dot{A}^{2} e^{2 A-2 B}\right] \\
R_{\nu \sigma}= & \alpha_{E}\left[\left(\eta_{\nu \sigma}-\delta_{\sigma}^{0} \eta_{\nu 0}\right)(\ddot{A}-\dot{A} \dot{B}) e^{2 A-2 B}\right. \\
& -(D-1) \delta_{\nu}^{0} \delta_{\sigma}^{0}\left(\ddot{A}+\dot{A}^{2}-\dot{A} \dot{B}-\dot{A}^{2} e^{2 A-2 B}\right) \\
& \left.+(D-1) \eta_{\nu \sigma} \dot{A}^{2} e^{2 A-2 B}\right], \\
R= & e^{-2 B} \alpha_{E}\left[2(D-1)(\ddot{A}-\dot{A} \dot{B})+D(D-1) \dot{A}^{2}\right]
\end{aligned}
$$

where in the application to the original model one should set $D=10$.

The field equations of the gravitino and the dilatino that follow from the Lagrangian of eq. (2.18) are

$$
\begin{aligned}
\Gamma^{\mu \rho \nu} D_{\rho} \psi_{\nu}+\frac{1}{2 \sqrt{2}} \Gamma^{\nu} \Gamma^{\mu} \lambda \partial_{\nu} \phi & =0, \\
\Gamma^{\mu} D_{\mu} \lambda+\frac{1}{2 \sqrt{2}} \Gamma^{\mu} \Gamma^{\nu} \psi_{\mu} \partial_{\nu} \phi & =0
\end{aligned}
$$

and are to be combined with the constraint of eq. (2.19).

Let us now try to find a solution with vanishing dilatino $\lambda=0$. In this case, the field equation of the gravitino becomes

$$
\Gamma^{\mu \rho \nu} D_{\rho} \psi_{\nu}=0
$$


with the constraints $\Gamma^{\mu} \psi_{\mu}=0$ from eq. (2.19) and $\partial_{\mu} \phi \psi^{\mu}=0$, which follow from the field equation of the dilatino. Applying $\Gamma_{\mu}$ to the field equation gives the additional constraint $D_{\mu} \psi^{\mu}=0$, while the covariant divergence of the field equation gives further additional constraint $R_{\mu \nu} \Gamma^{\nu} \psi^{\mu}=0$.

It is simple to see that all of these four constraints are solved by $\psi_{0}=0$ and $\Gamma^{i} \psi_{i}=0$, using the explicit forms of the Christoffel symbols, the spin connection and the Ricci tensor. Therefore, the problem is reduces to solve the field equation

$$
\Gamma^{\mu} D_{\mu} \psi_{i}=0
$$

where the field is subject to the constraint $\Gamma^{i} \psi_{i}=0$. The explicit form of the second order equation $\Gamma^{\nu} D_{\nu} \Gamma^{\mu} D_{\mu} \psi_{i}=0$ is

$$
\ddot{\psi}_{i}+\left(7 \dot{A}+\frac{3}{4} \dot{\phi}\right) \dot{\psi}_{i}+\frac{49}{4} \dot{A}^{2} \psi_{i}-\frac{7}{32} \dot{\phi}^{2} \psi_{i}=0
$$

but rescaling the field according to

$$
\psi_{i} \longrightarrow \Omega^{w_{3 / 2}} \psi_{i}
$$

it takes a very simple form:

$$
\ddot{\psi}_{i}+\frac{3}{4} \dot{\phi} \dot{\psi}_{i}=0
$$

Using the explicit expression of the background in eq. (4.24), the field equation reduces to

$$
\frac{d^{2} \psi_{i}}{d \tau^{2}}+\frac{3}{4}\left(\frac{2}{3 \tau}-\frac{3 \tau}{2}\right) \frac{d \psi_{i}}{d \tau}=0
$$

whose solution can be expressed in terms of the incomplete $\Gamma$-function, according to

$$
\psi_{i}=C_{i}^{(1)}+C_{i}^{(2)} \Gamma\left(\frac{1}{4},-\frac{9}{16} \tau^{2}\right),
$$

where $C_{i}^{(1)}$ and $C_{i}^{(2)}$ are integration constants that satisfy the conditions $\Gamma^{i} C_{i}^{(1)}=0$ and $\Gamma^{i} C_{i}^{(2)}=0$. They key point is that these solutions are not oscillatory. Moreover, if we require a finite $\psi_{i}$ at $\tau \rightarrow \infty$, the only option is

$$
\psi_{i}=C_{i}^{(1)}
$$

with $\Gamma^{i} C_{i}^{(1)}=0$, which points again to a massless gravitino in view of our new criterion, namely no "rest mass" or no contribution to the energy density $T_{00}$. In this respect, we can conclude that the gravitino is massless in this cosmological vacuum, which somehow replaces flat spacetime in the Sugimoto model.

The next issue concerns the actual number of degrees of freedom that are carried by the gravitino in the unitary gauge. To begin with, a Majorana vector-spinor field $\psi_{\mu}$ has $D \times 2^{[D / 2]}$ real degrees of freedom, or 320 in $D=10$. Since $\psi^{0}=0$ and $\Gamma^{i} \psi_{i}=0$, this number is readily cut to 256 in $D=10$. Moreover, $\psi_{\mu}$ is also a Weyl field, so that number 
is again reduced by a factor of two, or to 128 degrees of freedom in $D=10$. As usual, the first order Dirac equation that is left in unitary gauge

$$
\Gamma^{\rho} D_{\rho} \psi_{\mu}=0
$$

amounts, in our background, to a condition $\gamma^{0} \psi_{\mu}=0$, and considering projection operators $P_{ \pm}=\left(1 \pm i \gamma^{0}\right) / 2$, one is finally left with 64 degrees of freedom. This number results precisely from the 56 degrees of freedom carried by the original gravitino and the 8 degrees of freedom carried by the Nambu-Goldstone fermion.

To reiterate, the gravitino in the Sugimoto model behaves as a massless field in its cosmological background of $[60,61]$, although it combines the degrees of freedom that a massless gravitino would describe in a flat spacetime with those of the absorbed NambuGoldstone fermion. The two fields recover separate lives, consistently with this interpretation and known facts, if one turns off the tadpole potential. Moreover, this number is the half of the degrees of freedom that a massive spin- $3 / 2$ field would have in a tendimensional Minkowski spacetime, as demanded by the orientifold projection that underlies the Sugimoto model.

\section{Conclusions}

We have investigated the behavior of the gravitino in a class of orientifold models [42-51] with "brane supersymmetry breaking" [52-57], where supersymmetry is non-linearly realized while a gravitino mass term is not allowed. These models require non-trivial spacetime backgrounds, so that the notion of mass entails some subtleties and is different from the more familiar case of a flat spacetime. In a de Sitter spacetime null-cone propagation in the symmetric coordinate system provides an accepted criterion of masslessness, and along these lines we have proposed a new criterion for fermions that applies in the flat slicing coordinate system. The new criterion rests on a cosmological interpretation, and applies to more general background spacetimes. These include the spatially flat geometries where "brane supersymmetry breaking" brings along the climbing mechanism [75-77]. This could provide the initial impulse to start an inflationary phase [79-91], and with a short inflation this type of dynamics could have had some bearing on the low- $\ell$ CMB anomalies [112-115].

We have investigated the gravitino mass in the Sugimoto model, with reference to the background field configuration of [75-77], which is more complicated than de Sitter spacetime, arriving at conclusions that are reasonable with a massless gravitino. The overall lesson is consistent with massless gravitinos in non-trivial backgrounds that entail different numbers of degrees of freedom than in flat spacetime. Let us also stress that the "cosmological" criterion of masslessness in de Sitter-like backgrounds also applies to a conformal scalar field theory with non-minimal coupling to the scalar curvature, consistently with our view. Even in this case, the field equation for a suitably rescaled scalar field allows non-oscillatory solutions for which the energy density vanishes, although there is also a different option, which is proportional to $a^{-D}$, where $a=\exp \left(H x^{0}\right)$ is the scale factor. The latter contribution is typical of massless radiation. 
There is a host of evidence that our Universe has never been exactly a flat Minkowski spacetime, and that now it is close to a de Sitter spacetime. Fields with unusual numbers of degrees of freedom can thus be of interest, in principle, for Cosmology. A recent investigation along these lines can be found in [116-119], for example.

\section{Acknowledgments}

The author would like to thank S. Ferrara for helpful discussions and A. Sagnotti for helpful discussions, suggestions and a careful reading of the manuscript. The author would also like to thank Scuola Normale Superiore for the kind hospitality and for partial support while this work was in progress.

Open Access. This article is distributed under the terms of the Creative Commons Attribution License (CC-BY 4.0), which permits any use, distribution and reproduction in any medium, provided the original author(s) and source are credited.

\section{References}

[1] P. Ramond, Dual Theory for Free Fermions, Phys. Rev. D 3 (1971) 2415 [inSPIRE].

[2] Yu. A. Golfand and E.P. Likhtman, Extension of the Algebra of Poincaré Group Generators and Violation of p Invariance, JETP Lett. 13 (1971) 323 [Pisma Zh. Eksp. Teor. Fiz. 13 (1971) 452]. [INSPIRE].

[3] J.-L. Gervais and B. Sakita, Field Theory Interpretation of Supergauges in Dual Models, Nucl. Phys. B 34 (1971) 632 [inSPIRE].

[4] J. Wess and B. Zumino, A Lagrangian Model Invariant Under Supergauge Transformations, Phys. Lett. B 49 (1974) 52 [INSPIRE].

[5] J. Wess and B. Zumino, Supergauge Transformations in Four-Dimensions, Nucl. Phys. B 70 (1974) 39 [INSPIRE].

[6] A. Salam and J.A. Strathdee, Supergauge Transformations, Nucl. Phys. B 76 (1974) 477 [INSPIRE].

[7] S. Ferrara and B. Zumino, Supergauge Invariant Yang-Mills Theories, Nucl. Phys. B 79 (1974) 413 [INSPIRE].

[8] A. Salam and J.A. Strathdee, Supersymmetry and Nonabelian Gauges, Phys. Lett. B 51 (1974) 353 [INSPIRE].

[9] P. Fayet and J. Iliopoulos, Spontaneously Broken Supergauge Symmetries and Goldstone Spinors, Phys. Lett. B 51 (1974) 461 [INSPIRE].

[10] D.Z. Freedman, P. van Nieuwenhuizen and S. Ferrara, Progress Toward a Theory of Supergravity, Phys. Rev. D 13 (1976) 3214 [InSPIRE].

[11] S. Deser and B. Zumino, Consistent Supergravity, Phys. Lett. B 62 (1976) 335 [inSPIRE].

[12] D.Z. Freedman and A. Van Proeyen, Supergravity, Cambridge University Press, Cambridge U.K. (2012).

[13] P. Van Nieuwenhuizen, Supergravity, Phys. Rept. 68 (1981) 189 [InSPIRE]. 
[14] S. Ferrara and A. Sagnotti, Supergravity at 40: Reflections and Perspectives, Riv. Nuovo Cim. 40 (2017) 1 [J. Phys. Conf. Ser. 873 (2017) 012014] [arXiv:1702.00743] [InSPIRE].

[15] M.B. Green, J.H. Schwarz and E. Witten, Superstring Theory, 2 volumes, Cambridge University Press, Cambridge U.K. (1987).

[16] J. Polchinski, String theory, 2 volumes, Cambridge University Press, Cambridge U.K. (1998).

[17] C.V. Johnson, D-branes, Cambridge University Press, Cambridge U.K. (2003).

[18] B. Zwiebach, A first course in string theory, Cambridge University Press, Cambridge U.K. (2004).

[19] K. Becker, M. Becker and J.H. Schwarz, String theory and M-theory: A modern introduction, Cambridge University Press, Cambridge U.K. (2007).

[20] E. Kiritsis, String theory in a nutshell, Cambridge University Press, Cambridge U.K. (2007).

[21] P. West, Introduction to strings and branes, Cambridge University Press, Cambridge U.K. (2012).

[22] D.V. Volkov and V.P. Akulov, Is the Neutrino a Goldstone Particle?, Phys. Lett. B 46 (1973) 109 [INSPIRE].

[23] D.V. Volkov and V.A. Soroka, Higgs Effect for Goldstone Particles with Spin 1/2, JETP Lett. 18 (1973) 312 [Pisma Zh. Eksp. Teor. Fiz. 18 (1973) 529] [INSPIRE].

[24] D.V. Volkov and V.A. Soroka, Gauge fields for symmetry group with spinor parameters, Theor. Math. Phys. 20 (1974) 829 [Teor. Mat. Fiz. 20 (1974) 291] [inSPIRE].

[25] S. Deser and B. Zumino, Broken Supersymmetry and Supergravity, Phys. Rev. Lett. 38 (1977) 1433 [INSPIRE].

[26] E. Dudas, S. Ferrara, A. Kehagias and A. Sagnotti, Properties of Nilpotent Supergravity, JHEP 09 (2015) 217 [arXiv: 1507.07842] [INSPIRE].

[27] F. Hasegawa and Y. Yamada, Component action of nilpotent multiplet coupled to matter in 4 dimensional $\mathcal{N}=1$ supergravity, JHEP 10 (2015) 106 [arXiv:1507.08619] [INSPIRE].

[28] E.A. Bergshoeff, D.Z. Freedman, R. Kallosh and A. Van Proeyen, Pure de Sitter Supergravity, Phys. Rev. D 92 (2015) 085040 [Erratum ibid. D 93 (2016) 069901] [arXiv: 1507.08264] [INSPIRE].

[29] N. Cribiori, G. Dall'Agata and F. Farakos, From Linear to Non-linear SUSY and Back Again, JHEP 08 (2017) 117 [arXiv:1704.07387] [INSPIRE].

[30] R. Casalbuoni, S. De Curtis, D. Dominici, F. Feruglio and R. Gatto, Nonlinear Realization of Supersymmetry Algebra From Supersymmetric Constraint, Phys. Lett. B 220 (1989) 569 [INSPIRE].

[31] A. Brignole, F. Feruglio and F. Zwirner, On the effective interactions of a light gravitino with matter fermions, JHEP 11 (1997) 001 [hep-th/9709111] [INSPIRE].

[32] Z. Komargodski and N. Seiberg, From Linear SUSY to Constrained Superfields, JHEP 09 (2009) 066 [arXiv: 0907.2441] [InSPIRE].

[33] S.M. Kuzenko and S.J. Tyler, Relating the Komargodski-Seiberg and Akulov-Volkov actions: Exact nonlinear field redefinition, Phys. Lett. B 698 (2011) 319 [arXiv:1009.3298] [INSPIRE]. 
[34] I. Bandos, L. Martucci, D. Sorokin and M. Tonin, Brane induced supersymmetry breaking and de Sitter supergravity, JHEP 02 (2016) 080 [arXiv: 1511.03024] [INSPIRE].

[35] I. Bandos, M. Heller, S.M. Kuzenko, L. Martucci and D. Sorokin, The Goldstino brane, the constrained superfields and matter in $\mathcal{N}=1$ supergravity, JHEP 11 (2016) 109 [arXiv: 1608.05908] [INSPIRE].

[36] E. Cremmer, B. Julia, J. Scherk, S. Ferrara, L. Girardello and P. van Nieuwenhuizen, Spontaneous Symmetry Breaking and Higgs Effect in Supergravity Without Cosmological Constant, Nucl. Phys. B 147 (1979) 105 [inSPIRE].

[37] A.H. Chamseddine, R.L. Arnowitt and P. Nath, Locally Supersymmetric Grand Unification, Phys. Rev. Lett. 49 (1982) 970 [INSPIRE].

[38] J. Bagger and E. Witten, The Gauge Invariant Supersymmetric Nonlinear $\sigma$-model, Phys. Lett. B 118 (1982) 103 [INSPIRE].

[39] E. Cremmer, S. Ferrara, L. Girardello and A. Van Proeyen, Yang-Mills Theories with Local Supersymmetry: Lagrangian, Transformation Laws and SuperHiggs Effect, Nucl. Phys. B 212 (1983) 413 [INSPIRE].

[40] E. Cremmer, S. Ferrara, C. Kounnas and D.V. Nanopoulos, Naturally Vanishing Cosmological Constant in $N=1$ Supergravity, Phys. Lett. B 133 (1983) 61 [InSPIRE].

[41] S.B. Giddings, S. Kachru and J. Polchinski, Hierarchies from fluxes in string compactifications, Phys. Rev. D 66 (2002) 106006 [hep-th/0105097] [INSPIRE].

[42] A. Sagnotti, Open Strings and their Symmetry Groups, in Cargese '87, Non-Perturbative Quantum Field Theory, G. Mack et al. eds., Pergamon Press (1988), p. 521 [hep-th/0208020] [INSPIRE].

[43] G. Pradisi and A. Sagnotti, Open String Orbifolds, Phys. Lett. B 216 (1989) 59 [InSPIRE].

[44] P. Hořava, Strings on World Sheet Orbifolds, Nucl. Phys. B 327 (1989) 461 [InSPIRE].

[45] P. Hořava, Background Duality of Open String Models, Phys. Lett. B 231 (1989) 251 [INSPIRE].

[46] M. Bianchi and A. Sagnotti, On the systematics of open string theories, Phys. Lett. B 247 (1990) 517 [INSPIRE].

[47] M. Bianchi and A. Sagnotti, Twist symmetry and open string Wilson lines, Nucl. Phys. B 361 (1991) 519 [INSPIRE].

[48] M. Bianchi, G. Pradisi and A. Sagnotti, Toroidal compactification and symmetry breaking in open string theories, Nucl. Phys. B 376 (1992) 365 [INSPIRE].

[49] A. Sagnotti, A Note on the Green-Schwarz mechanism in open string theories, Phys. Lett. B 294 (1992) 196 [hep-th/9210127] [INSPIRE].

[50] E. Dudas, Theory and phenomenology of type-I strings and M-theory, Class. Quant. Grav. 17 (2000) R41 [hep-ph/0006190] [INSPIRE].

[51] C. Angelantonj and A. Sagnotti, Open strings, Phys. Rept. 371 (2002) 1 [Erratum ibid. 376 (2003) 339] [hep-th/0204089] [INSPIRE].

[52] S. Sugimoto, Anomaly cancellations in type-I D-9-D $\bar{D}-9$ system and the $\mathrm{USp}(32)$ string theory, Prog. Theor. Phys. 102 (1999) 685 [hep-th/9905159] [InSPIRE]. 
[53] I. Antoniadis, E. Dudas and A. Sagnotti, Brane supersymmetry breaking, Phys. Lett. B 464 (1999) 38 [hep-th/9908023] [INSPIRE].

[54] C. Angelantonj, Comments on open string orbifolds with a nonvanishing B(ab), Nucl. Phys. B 566 (2000) 126 [hep-th/9908064] [INSPIRE].

[55] G. Aldazabal and A.M. Uranga, Tachyon free nonsupersymmetric type IIB orientifolds via Brane - anti-brane systems, JHEP 10 (1999) 024 [hep-th/9908072] [INSPIRE].

[56] C. Angelantonj, I. Antoniadis, G. D'Appollonio, E. Dudas and A. Sagnotti, Type I vacua with brane supersymmetry breaking, Nucl. Phys. B 572 (2000) 36 [hep-th/9911081] [INSPIRE].

[57] J. Mourad and A. Sagnotti, An Update on Brane Supersymmetry Breaking, arXiv: 1711.11494 [INSPIRE].

[58] E. Dudas and J. Mourad, Consistent gravitino couplings in nonsupersymmetric strings, Phys. Lett. B 514 (2001) 173 [hep-th/0012071] [INSPIRE].

[59] G. Pradisi and F. Riccioni, Geometric couplings and brane supersymmetry breaking, Nucl. Phys. B 615 (2001) 33 [hep-th/0107090] [InSPIRE].

[60] E. Dudas and J. Mourad, Brane solutions in strings with broken supersymmetry and dilaton tadpoles, Phys. Lett. B 486 (2000) 172 [hep-th/0004165] [InSPIRE].

[61] R. Blumenhagen and A. Font, Dilaton tadpoles, warped geometries and large extra dimensions for nonsupersymmetric strings, Nucl. Phys. B 599 (2001) 241 [hep-th/0011269] [INSPIRE].

[62] S. Deser and R.I. Nepomechie, Gauge Invariance Versus Masslessness in de Sitter Space, Annals Phys. 154 (1984) 396 [InSPIRE].

[63] S. Deser and A. Waldron, Null propagation of partially massless higher spins in (A)dS and cosmological constant speculations, Phys. Lett. B 513 (2001) 137 [hep-th/0105181] [INSPIRE].

[64] S. Deser and A. Waldron, Gauge invariances and phases of massive higher spins in (A)dS, Phys. Rev. Lett. 87 (2001) 031601 [hep-th/0102166] [INSPIRE].

[65] T. Garidi, What is mass in de Sitterian physics?, hep-th/0309104 [INSPIRE].

[66] S. Deser and A. Waldron, Conformal invariance of partially massless higher spins, Phys. Lett. B 603 (2004) 30 [hep-th/0408155] [INSPIRE].

[67] F. Gursey and T.D. Lee, Spin 1/2 Wave Equation in de Sitter Space, Proc. Nat. Acad. Sci. 49 (1963) 179 [inSPIRE].

[68] Y. Tanii, Introduction to supergravity, SpringerBriefs in Mathematical Physics (2014).

[69] D.Z. Freedman, Supergravity with Axial Gauge Invariance, Phys. Rev. D 15 (1977) 1173 [INSPIRE].

[70] B. Zumino, Nonlinear Realization of Supersymmetry in de Sitter Space, Nucl. Phys. B 127 (1977) 189 [INSPIRE].

[71] S. Ferrara and A. Van Proeyen, Mass Formulae for Broken Supersymmetry in Curved Space-Time, Fortsch. Phys. 64 (2016) 896 [arXiv: 1609.08480] [INSPIRE].

[72] A.O. Barut and B.-W. Xu, Conformal Covariance and the Probability Interpretation of Wave Equations, Phys. Lett. A 82 (1981) 218 [inSPIRE]. 
[73] S. Deser and A. Waldron, Partial masslessness of higher spins in (A)dS, Nucl. Phys. B 607 (2001) 577 [hep-th/0103198] [INSPIRE].

[74] J.G. Russo, Exact solution of scalar tensor cosmology with exponential potentials and transient acceleration, Phys. Lett. B 600 (2004) 185 [hep-th/0403010] [INSPIRE].

[75] E. Dudas, N. Kitazawa and A. Sagnotti, On Climbing Scalars in String Theory, Phys. Lett. B 694 (2011) 80 [arXiv: 1009.0874] [INSPIRE].

[76] A. Sagnotti, Brane SUSY breaking and inflation: implications for scalar fields and CMB distortion, Phys. Part. Nucl. Lett. 11 (2014) 836 [arXiv:1303.6685] [INSPIRE].

[77] P. Fré, A. Sagnotti and A.S. Sorin, Integrable Scalar Cosmologies I. Foundations and links with String Theory, Nucl. Phys. B 877 (2013) 1028 [arXiv:1307.1910] [InSPIRE].

[78] C. Condeescu and E. Dudas, Kasner solutions, climbing scalars and big-bang singularity, JCAP 08 (2013) 013 [arXiv:1306.0911] [InSPIRE].

[79] A.A. Starobinsky, A New Type of Isotropic Cosmological Models Without Singularity, Phys. Lett. B 91 (1980) 99 [INSPIRE].

[80] D. Kazanas, Dynamics of the Universe and Spontaneous Symmetry Breaking, Astrophys. J. 241 (1980) L59 [INSPIRE].

[81] K. Sato, Cosmological Baryon Number Domain Structure and the First Order Phase Transition of a Vacuum, Phys. Lett. B 99 (1981) 66 [INSPIRE].

[82] A.H. Guth, The Inflationary Universe: A Possible Solution to the Horizon and Flatness Problems, Phys. Rev. D 23 (1981) 347 [inSPIRE].

[83] A.D. Linde, A New Inflationary Universe Scenario: A Possible Solution of the Horizon, Flatness, Homogeneity, Isotropy and Primordial Monopole Problems, Phys. Lett. B 108 (1982) 389 [INSPIRE].

[84] A. Albrecht and P.J. Steinhardt, Cosmology for Grand Unified Theories with Radiatively Induced Symmetry Breaking, Phys. Rev. Lett. 48 (1982) 1220 [inSPIRE].

[85] A.D. Linde, Chaotic Inflation, Phys. Lett. B 129 (1983) 177 [InSPIRE].

[86] N. Bartolo, E. Komatsu, S. Matarrese and A. Riotto, Non-Gaussianity from inflation: Theory and observations, Phys. Rept. 402 (2004) 103 [astro-ph/0406398] [INSPIRE].

[87] V. Mukhanov, Physical foundations of cosmology, Cambridge University Press, Cambridge U.K. (2005).

[88] S. Weinberg, Cosmology, Cambridge University Press, Cambridge U.K. (2008).

[89] D.H. Lyth and A.R. Liddle, The primordial density perturbation: Cosmology, inflation and the origin of structure, Cambridge University Press, Cambridge U.K. (2009).

[90] D.S. Gorbunov and V.A. Rubakov, Introduction to the theory of the early universe: Cosmological perturbations and inflationary theory, World Scientific, Hackensack U.S.A. (2011).

[91] J. Martin, C. Ringeval and V. Vennin, Encyclopæedia Inflationaris, Phys. Dark Univ. 5-6 (2014) 75 [arXiv:1303.3787] [INSPIRE].

[92] A.D. Linde, A Toy model for open inflation, Phys. Rev. D 59 (1999) 023503 [hep-ph/9807493] [INSPIRE]. 
[93] C.R. Contaldi, M. Peloso, L. Kofman and A.D. Linde, Suppressing the lower multipoles in the CMB anisotropies, JCAP 07 (2003) 002 [astro-ph/0303636] [INSPIRE].

[94] Y.-S. Piao, B. Feng and X.-m. Zhang, Suppressing CMB quadrupole with a bounce from contracting phase to inflation, Phys. Rev. D 69 (2004) 103520 [hep-th/0310206] [INSPIRE].

[95] Y.-S. Piao, A Possible explanation to low CMB quadrupole, Phys. Rev. D 71 (2005) 087301 [astro-ph/0502343] [INSPIRE].

[96] D. Boyanovsky, H.J. de Vega and N.G. Sanchez, CMB quadrupole suppression. 2. The early fast roll stage, Phys. Rev. D 74 (2006) 123007 [astro-ph/0607487] [INSPIRE].

[97] C. Destri, H.J. de Vega and N.G. Sanchez, The CMB Quadrupole depression produced by early fast-roll inflation: $M C M C$ analysis of WMAP and SDSS data, Phys. Rev. D 78 (2008) 023013 [arXiv:0804.2387] [INSPIRE].

[98] F.J. Cao, H.J. de Vega and N.G. Sanchez, Quantum slow-roll and quantum fast-roll inflationary initial conditions: CMB quadrupole suppression and further effects on the low CMB multipoles, Phys. Rev. D 78 (2008) 083508 [arXiv:0809.0623] [INSPIRE].

[99] R.K. Jain, P. Chingangbam, J.-O. Gong, L. Sriramkumar and T. Souradeep, Punctuated inflation and the low CMB multipoles, JCAP 01 (2009) 009 [arXiv:0809.3915] [INSPIRE].

[100] E. Ramirez and D.J. Schwarz, $\phi^{4}$ inflation is not excluded, Phys. Rev. D 80 (2009) 023525 [arXiv:0903.3543] [INSPIRE].

[101] E. Ramirez and D.J. Schwarz, Predictions of just-enough inflation, Phys. Rev. D 85 (2012) 103516 [arXiv: 1111.7131] [InSPIRE].

[102] R.K. Jain, P. Chingangbam, L. Sriramkumar and T. Souradeep, The tensor-to-scalar ratio in punctuated inflation, Phys. Rev. D 82 (2010) 023509 [arXiv:0904.2518] [INSPIRE].

[103] C. Destri, H.J. de Vega and N.G. Sanchez, The pre-inflationary and inflationary fast-roll eras and their signatures in the low CMB multipoles, Phys. Rev. D 81 (2010) 063520 [arXiv: 0912.2994] [INSPIRE].

[104] E. Ramirez, Low power on large scales in just enough inflation models, Phys. Rev. D 85 (2012) 103517 [arXiv:1202.0698] [INSPIRE].

[105] Z.-G. Liu, Z.-K. Guo and Y.-S. Piao, Obtaining the CMB anomalies with a bounce from the contracting phase to inflation, Phys. Rev. D 88 (2013) 063539 [arXiv:1304.6527] [INSPIRE].

[106] F.G. Pedro and A. Westphal, Low- $\ell$ CMB power loss in string inflation, JHEP 04 (2014) 034 [arXiv: 1309.3413] [INSPIRE].

[107] M. Cicoli, S. Downes, B. Dutta, F.G. Pedro and A. Westphal, Just enough inflation: power spectrum modifications at large scales, JCAP 12 (2014) 030 [arXiv:1407.1048] [INSPIRE].

[108] R. Bousso, D. Harlow and L. Senatore, Inflation after False Vacuum Decay, Phys. Rev. D 91 (2015) 083527 [arXiv: 1309.4060] [InSPIRE].

[109] Z.-G. Liu, Z.-K. Guo and Y.-S. Piao, CMB anomalies from an inflationary model in string theory, Eur. Phys. J. C 74 (2014) 3006 [arXiv:1311.1599] [INSPIRE].

[110] A.Y. Kamenshchik, A. Tronconi and G. Venturi, Quantum Gravity and the Large Scale Anomaly, JCAP 04 (2015) 046 [arXiv:1501.06404] [INSPIRE]. 
[111] Y.-F. Cai, E.G.M. Ferreira, B. Hu and J. Quintin, Searching for features of a string-inspired inflationary model with cosmological observations, Phys. Rev. D 92 (2015) 121303 [arXiv: 1507.05619] [INSPIRE].

[112] E. Dudas, N. Kitazawa, S.P. Patil and A. Sagnotti, CMB Imprints of a Pre-Inflationary Climbing Phase, JCAP 05 (2012) 012 [arXiv:1202.6630] [INSPIRE].

[113] A. Gruppuso and A. Sagnotti, Observational Hints of a Pre-Inflationary Scale?, Int. J. Mod. Phys. D 24 (2015) 1544008 [arXiv:1506.08093] [InSPIRE].

[114] A. Gruppuso, N. Kitazawa, N. Mandolesi, P. Natoli and A. Sagnotti, Pre-Inflationary Relics in the CMB?, Phys. Dark Univ. 11 (2016) 68 [arXiv:1508.00411] [INSPIRE].

[115] A. Gruppuso, N. Kitazawa, M. Lattanzi, N. Mandolesi, P. Natoli and A. Sagnotti, The Evens and Odds of CMB Anomalies, Phys. Dark Univ. 20 (2018) 49 [arXiv:1712.03288] [INSPIRE].

[116] A. Kehagias and A. Riotto, On the Inflationary Perturbations of Massive Higher-Spin Fields, JCAP 07 (2017) 046 [arXiv:1705.05834] [INSPIRE].

[117] N. Bartolo, A. Kehagias, M. Liguori, A. Riotto, M. Shiraishi and V. Tansella, Detecting higher spin fields through statistical anisotropy in the CMB and galaxy power spectra, Phys. Rev. D 97 (2018) 023503 [arXiv: 1709.05695] [INSPIRE].

[118] D. Baumann, G. Goon, H. Lee and G.L. Pimentel, Partially Massless Fields During Inflation, arXiv: 1712.06624 [INSPIRE].

[119] G. Franciolini, A. Kehagias and A. Riotto, Imprints of Spinning Particles on Primordial Cosmological Perturbations, JCAP 02 (2018) 023 [arXiv: 1712.06626] [INSPIRE]. 